\title{
High ANXA7 Potentiates Eucalyptol Toxicity in Hormone-refractory Prostate Cancer
}

\author{
XIMENA LEIGHTON*, ALAKESH BERA*, OFER EIDELMAN, MICHAEL EKLUND, \\ NARAYANAN PUTHILLATHU, HARVEY B. POLLARD and MEERA SRIVASTAVA \\ Department of Anatomy, Physiology, and Genetics, and Institute for Molecular Medicine, \\ Uniformed Services University School of Medicine (USUHS), Bethesda, MD, U.S.A.
}

\begin{abstract}
Background/Aim: Our studies showed that ANXA7 is a novel tumor suppressor gene that is lost in various aggressive forms of prostate cancer. However, little is known about the role of ANXA7 in the anticancer drug treatment towards different cancers. Materials and Methods: The expression of ANXA7 was measured in the 60 cancer cell lines of the NCI-60 ADS project and correlated with the enhanced sensitivity to over 30,000 natural and synthetic compounds. Results: Eucalyptol showed a high positive correlation with ANXA7 expression and castration-resistant prostate cancer cell death occurred very effectively in response to the combination of eucalyptol and overexpressed wt-ANXA7 than either agent alone. The synergistic effects of ANXA7 and eucalyptol resulted in concordant changes in gene expression profiles particularly of Ras family members, MDM4, NF- $\mathrm{B} B$ and VEGF. Conclusion: Overexpression of ANXA7 enhances eucalyptol cytotoxicity in prostate cancer cell lines.
\end{abstract}

Prostate cancer is one of the most common cancers and is now the second leading cause of cancer-associated death in men. In 2018 , it is estimated that an approximate 164,690 new cases and 29,430 prostate cancer-related deaths will occur in USA (1). Therapeutic modalities such as radical prostatectomy and radiotherapy are considered curative for localized disease. However, no treatments are available for metastatic castrationresistant prostate cancer (2). Androgen ablation is highly

This article is freely accessible online.

*These Authors contributed equally to this study.

Correspondence to: Meera Srivastava, Ph.D., Department of Anatomy, Physiology and Genetics, USU School of Medicine, 4301 Jones Bridge Road, Bethesda MD, 20814, U.S.A. Tel: +1 3012953204, Fax: +1 3012951786, e-mail: meera.srivastava@usuhs.edu

Key Words: Prostate cancer, eucalyptol, chemo-sensitivity, ANXA7, National Cancer Institute - 60 cancer cell line screening (NCI-60), Anticancer Drug Screen (NCI-60 ADS). effective in the treatment of castration-resistant prostate cancer because it triggers programmed cell death in castration-resistant prostate cancer cells. Unfortunately, the presence of castrationresistant prostate cancer cells within the tumor, which do not undergo apoptosis upon androgen withdraw, eventually cause relapse (3). Induction of apoptosis in sensitive tumor cells is the main mechanism of action of chemotherapy agents in human cancers. These castration-resistant prostate cancer cells are resistant to cytotoxic drugs due to their low rate of proliferation. Despite several hundred clinical studies of both experimental and approved chemotherapeutic agents, chemotherapy has limited antitumor activity, with an objective response rate of less than $10 \%$ and no demonstrated survival benefit (4). Thus, the key to overcoming chemotherapeutic resistance will be to combine current therapeutic strategies with novel mechanism-based therapies.

Cancer cells, in general, display decreased sensitivity to a wide variety of therapeutic agents, and oncogenes and tumor suppressor genes of cancer cells may be responsible for their preferential sensitivity to certain antineoplastic agents. We have found that the ANXA7 gene is defective in $35 \%$ of human prostate cancer specimens (5) and $40 \%$ of human breast cancer specimens (6). Remarkably, loss of expression of ANXA7 is associated with the development of androgen independence in prostate cancer. However, little is known about ANXA7 function regarding the pharmacological features of cancer cells.

The NCI-60 cell line panel has been used extensively in drug evaluation in the National Cancer Institute Anticancer Drug Screen (NCI-ADS) project. The NCI-60 panel consists of 60 human cancer cell lines used by the Developmental Therapeutics Program (DTS) of the National Cancer Institute (NCI) to screen over 100,000 chemical compounds for anticancer activity since $1990(7,8)$. Patterns of drug activity and molecular profiling of cells have yielded rich information on mechanisms of drug action and inhibition $(7,9,10)$. The NCI-60 cancer cell lines derived from leukemias, melanomas, and cancers of ovarian, breast, prostate, lung, renal, colon, and central nervous system origin. The mechanisms of the 


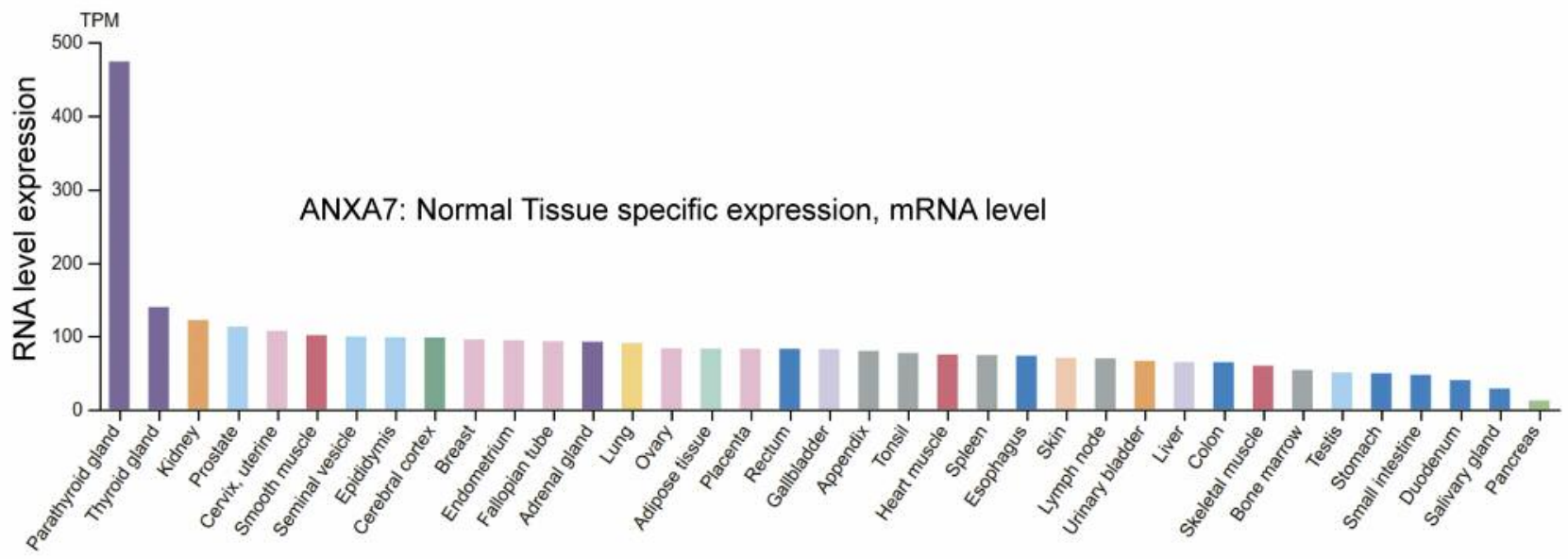

Figure 1. mRNA expression level of ANXA7 in normal tissue. Data extracted from Human Protein Atlas (HPA) RNA-seq project and RNA-seq data from the Genotype-Tissue Expression (GTEx) project. The RNA-seq tissue data is reported as mean TPM (transcripts per million), corresponding to mean values of the different individual samples from each tissue.

anticancer drug action and resistance are determined by the relevant information of the patterns of drug activity across the cell lines as well as patterns of cell sensitivity across the set of tested drugs (11). In addition to this pharmacological characterization, the NCI-60 cells have been more extensively profiled at the DNA, mRNA, protein, and functional levels than any other set of cells in existence.

Therefore, the interaction of anticancer agents with specific cellular targets can be identified by correlating target expression patterns with cytotoxicity patterns. Essentially, if we are able to measure the expression of ANXA7, it would be possible to link ANXA7 function to a variety of other molecular, physiological, and pharmacological features of the cells. The identification of new agents that are affected by the activity of ANXA7 was sought by correlating ANXA7 expression levels with the cytotoxicity of the 30,000 compounds and 170 known antineoplastic agents present in the National Cancer Institute (NCI) drug screen database. Eucalyptol was identified by the COMPARE program and was further tested against an independent set of tumor cell lines. The results of the present study revealed that overexpression of ANXA7 in the prostate cancer cell line correlated with enhanced sensitivity to eucalyptol. The ANXA7 regulated signaling pathway that provides sensitization to eucalyptol was examined using a genomic microarray platform to predict the mechanism. ANXA7-signaling pathways could be potentially important targets for anticancer therapy.

\section{Materials and Methods}

Microarray method for overall expression level measurements. The mRNA was isolated from logarithmically growing cells and the overall expression of a target gene (RNA-level) was calculated as described previously $(11,12)$. Briefly, labelled cDNA was prepared by reverse transcription of test cell mRNA in the presence of Cy5dUTP. Next, the reference probes were made by pooling equal amounts of mRNA from HL-60, K562, NCI-H226, COLO205, SNB-19, LOX-IMVI, OVCAR-3, OVCAR-4, CAKI-1, PC-3, MCF7, and Hs578T cell lines. The labeled reference cDNA was prepared from the pooled mRNA by reverse transcription in the presence of Cy3-dUTP. Test and reference probes were combined, denatured and hybridized overnight to Synteni microarrays containing cDNA from 9703 human clones. Arrays were scanned using a laser-scanning microscope $(11,12)$. The ScanAlyze program was used to analyze the microarray images and prepare data files for further analysis. The unit used for the final graphs is log (mRNA levels in cell line/mRNA levels in reference pool).

Drug activity screening. The drug profiling protocols of the NCI have been described (11). Briefly, the cells were grown in 96-well plates and exposed to the test compound for $48 \mathrm{~h}$. Growth inhibition was assessed by the sulpho-rhodamine $\mathrm{B}$ assay for cellular protein concentration and quantified as $\log \left(\mathrm{GI}_{50}\right)$, where $\mathrm{GI}_{50}$ is the concentration required to inhibit cell growth by $50 \%$ in comparison with untreated controls. The activity profile of a compound is composed of 60 such activity values, one for each cell line (13).

Interpretation of one or five-dose data. The one-dose data was presented as the mean graph of the percent growth of drug treated cells and similarly presented as mean graphs for 5-dose assay. The one-dose assay is growth relative to the control (no drug administration) and the number of cells at time zero (13). Therefore, it allows quantification of both growth inhibition (values between 0 and 100) and lethality (values less than 0 ). The calculation applied also for the 5-dose assay. For better understanding, a value of 100 means no growth inhibition and value of 40 would mean $60 \%$ growth inhibition. Similarly, value of 0 means no net growth and value of 40 would mean $40 \%$ lethality, with a particular drug over the course of the experiment. Finally, a value of -100 means all cells are dead. Information from the one-dose or five-dose mean graphs are available 
for COMPARE analysis. Compounds which exhibit significant growth inhibition in the one-dose Screen are then evaluated against the 60-cell panel at five concentration levels.

NCI-60 Data analysis. COMPARE software was used to assess whether the compounds present in the database displayed selective growth inhibition against the tumor cell lines with ANXA7 expression (14). Rank order correlations with a 50\% growth inhibitory pattern of response in the 60 cell lines were carried out against the database of unrestricted open compounds $(30,000)$ and 170 compounds including clinical drugs and agents that failed clinical trials and those where a mechanism of action has been defined. Pearson correlation coefficients and two tail $p$-values were included to help in assigning possible significance to these data. Drug responses or patterns of particular standard agents or open database compounds can be found at http://dtp.nci.nih.gov/docs/cancer/ searches/cancer_open_compounds.html. Information on Molecular Targets measured in the 60-cell panel can be found at http://dtp.nci.nih.gov/mtargets/mt_index.html.

Cell proliferation assay. Eucalyptol effects on growth inhibition were examined in a metastatic prostate cancer cell line (DU145) transfected with adenovirus vectors containing the wild-type ANXA7 gene. Initially, the cells were incubated with eucalyptol and 1,5 or 10 $\mathrm{pfu} / \mathrm{cell}$ of either control adenovirus, or adenovirus expressing wildtype ANXA7. Uninfected cells were analyzed in parallel. At preselected time intervals, the medium was aspirated. The cells were then incubated with $0.25 \mathrm{mg} / \mathrm{ml}$ of MTT for $1 \mathrm{~h}$ and then extracted with DMSO. The color change in the extract was measured at $550 \mathrm{~nm}$ by a spectrophotometer (Amersham, Piscataway, NJ, USA).

Preparation and labeling of RNA. Total RNAs from prostate cancer cells (DU145), either parental or transfected with either eucalyptol or ANXA7 were prepared and subjected to DNAse 1 digestion to eliminate genomic DNA contamination. Two rounds of purification of poly A+ RNA from total RNA were performed using the mRNA isolation kit from Invitrogen (Life Technologies, Carlsbad, CA, USA) as recommended by the manufacturer. The quality of the RNA was tested by running formaldehyde denatured agarose gel and quantitated by measuring the optical density at $260 \mathrm{~nm}$. A ${ }^{32} \mathrm{P}$ labeled cDNA probe was synthesized from $1 \mu \mathrm{g}$ of poly A+ RNA from the samples using MMLV reverse transcriptase, dNTP mix and CDS primer mix comprising the oligonucleotide sequences for the 1,200 cancer-related genes spotted on the atlas cDNA microarray. The reaction was carried out in a thermo-cycler set at $50^{\circ} \mathrm{C}$ for $25 \mathrm{~min}$ and terminated by the addition of $0.1 \mathrm{M}$ EDTA, $\mathrm{pH} 8.0$ and $1 \mathrm{mg} / \mathrm{ml}$ glycogen. The ${ }^{32} \mathrm{P}$ labeled cDNA probe was then purified from unincorporated ${ }^{32} \mathrm{P}$ labeled nucleotides by using a CHROMA SPIN-200 column (Takara Bio; Mountain View, CA, USA) as recommended by the manufacturer. The human atlas cDNA expression array containing 1200 cancer-related genes on a nylon membrane was pre-hybridized using Express Hyb (Takara Bio; Mountain View, CA, USA) at $68^{\circ} \mathrm{C}$ for one hour and hybridized overnight at $68^{\circ} \mathrm{C}$ with the denatured and neutralized ${ }^{32} \mathrm{P}$ labeled cDNA probe. The membrane was washed three times with $2 \mathrm{X}$ $\mathrm{SSC}, 1 \% \mathrm{SDS}$ at $68^{\circ} \mathrm{C}$ for 30 min each and twice with $0.1 \% \mathrm{SSC}, 0.5 \%$ SDS at $68^{\circ} \mathrm{C}$ for $30 \mathrm{~min}$ each. The atlas array was exposed overnight, and the results were compared with the known distribution of genes.

Imaging and quantitation of the cDNA microarray. Imaging data from the Storm PhosphorImager were downloaded into a Microsoft

\section{Level (relative to mean)}

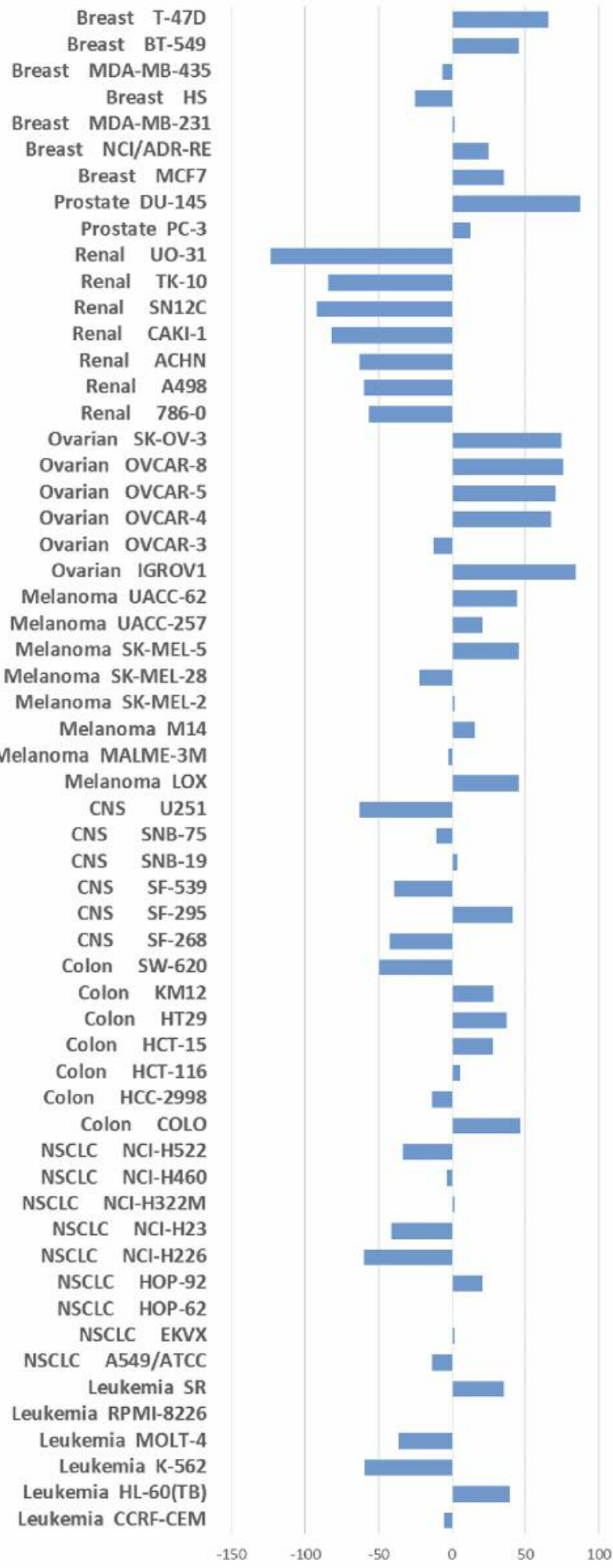

Figure 2. Correlation of ANXA7 gene expression profile in NCI-60 cancer cell lines panel. The mean expression level of ANXA7 gene expression was measured. 

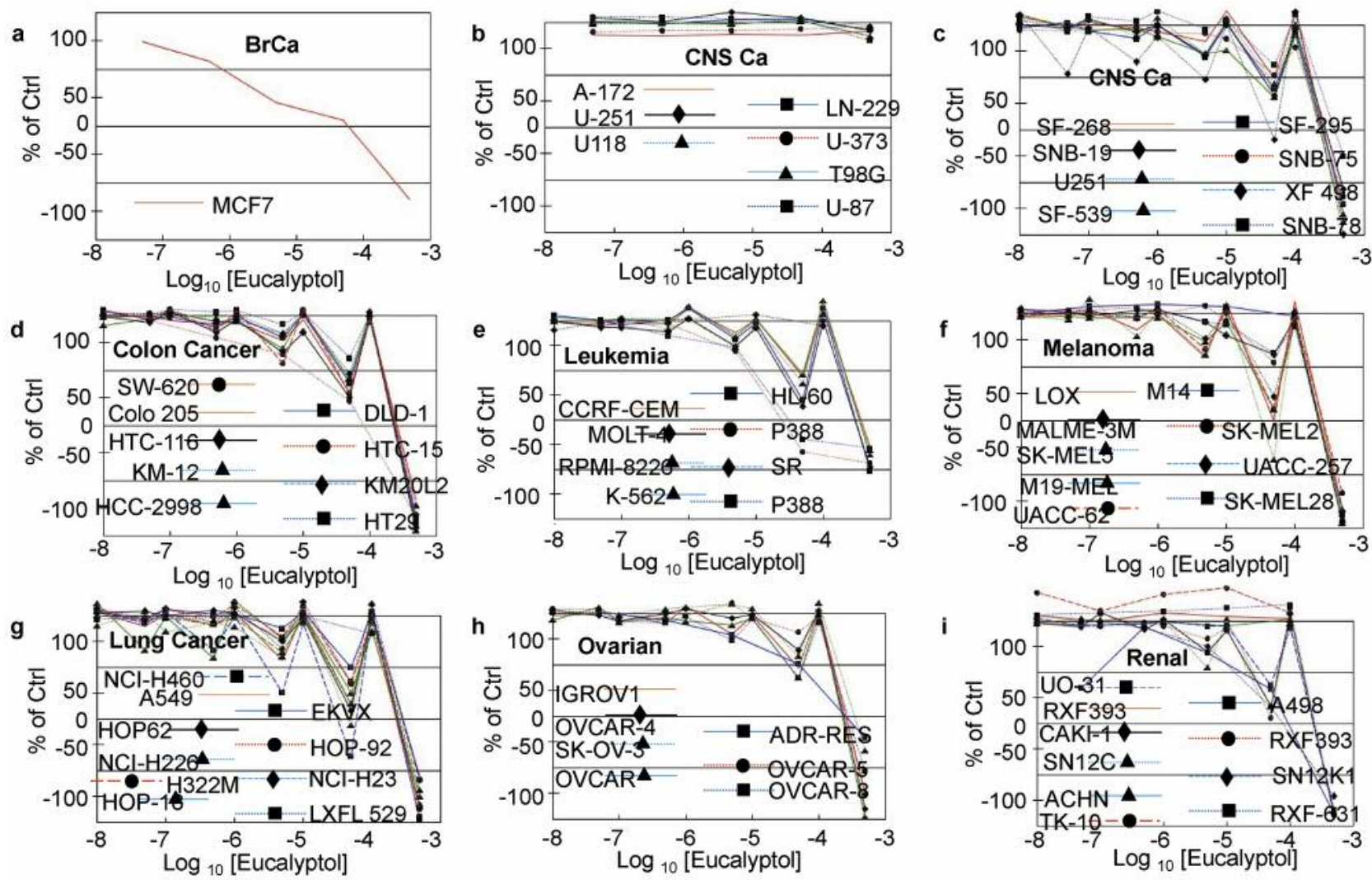

Figure 3. The effect of eucalyptol on different cell lines within the NIC-60 cell line panel. The dose (five dose experiment) response curves for eucalyptol (NCI drug ID: NSC6171). a-i. Dose response towards different cancer cell-type clusters.

Excel spreadsheet. Duplicate data points were averaged and equilibrated with the ubiquitin standard. Data was then analyzed using the Stanford University ScanAlyze software. These data points were also evaluated in parallel with the PSCAN program for point identification and with the JMP program for the graphical organization.

Statistical data mining from cDNA arrays. The first employed strategy is embodied in the GRASP methodology (Gene Ratio AnalysiS Paradigm) (15). The GRASP algorithm stipulates the changes in specific intensities of specific genes which are greater or less than an optimum standard deviation (S.D.) from the average changes of all genes in the entire array. This technique significantly increases the statistical power of the analysis.

\section{Results}

Correlation of anticancer compound activity with molecular target (ANXA7) measurements in the NCI-60 cancer cell lines. The developmental therapeutics (DTP) database at the National Cancer Institute consists of rank order correlations with $50 \%$ growth inhibitory pattern of drug response in the 60 cell lines. Since, the focus was to correlate the inhibitory effect of the anticancer drugs with the ANXA7 expression, the data was first extracted from the human protein atlas database for normal tissue level expression of ANXA7. The RNA-seq data indicates that the thyroid, kidney and prostate have the higher levels of ANXA7 gene expression (Figure 1). Next, the goal was to correlate patterns of anticancer compound activity with molecular target (ANXA7) measurements in the NCI 60 cancer cell lines. A positive correlation indicates that high levels of the target (or wildtype form) may be associated with sensitivity to the drug, while a negative correlation indicates that highlevels of the target (or wild-type form) confer cellular resistance to a given drug. Figure 2 shows the pattern of ANXA7 gene expression in NCI-60 tumor cell lines. This pattern is correlated with drug responses or patterns of particular standard agents (170 antineoplastic agents) or open database compounds $(30,000$ candidate drugs $)$ in the 60 cell lines. Preliminary studies identified that eucalyptol had the best positive Pearson correlative associations with ANXA7 indicating that eucalyptol sensitivity may be associated with overexpression of ANXA7. 


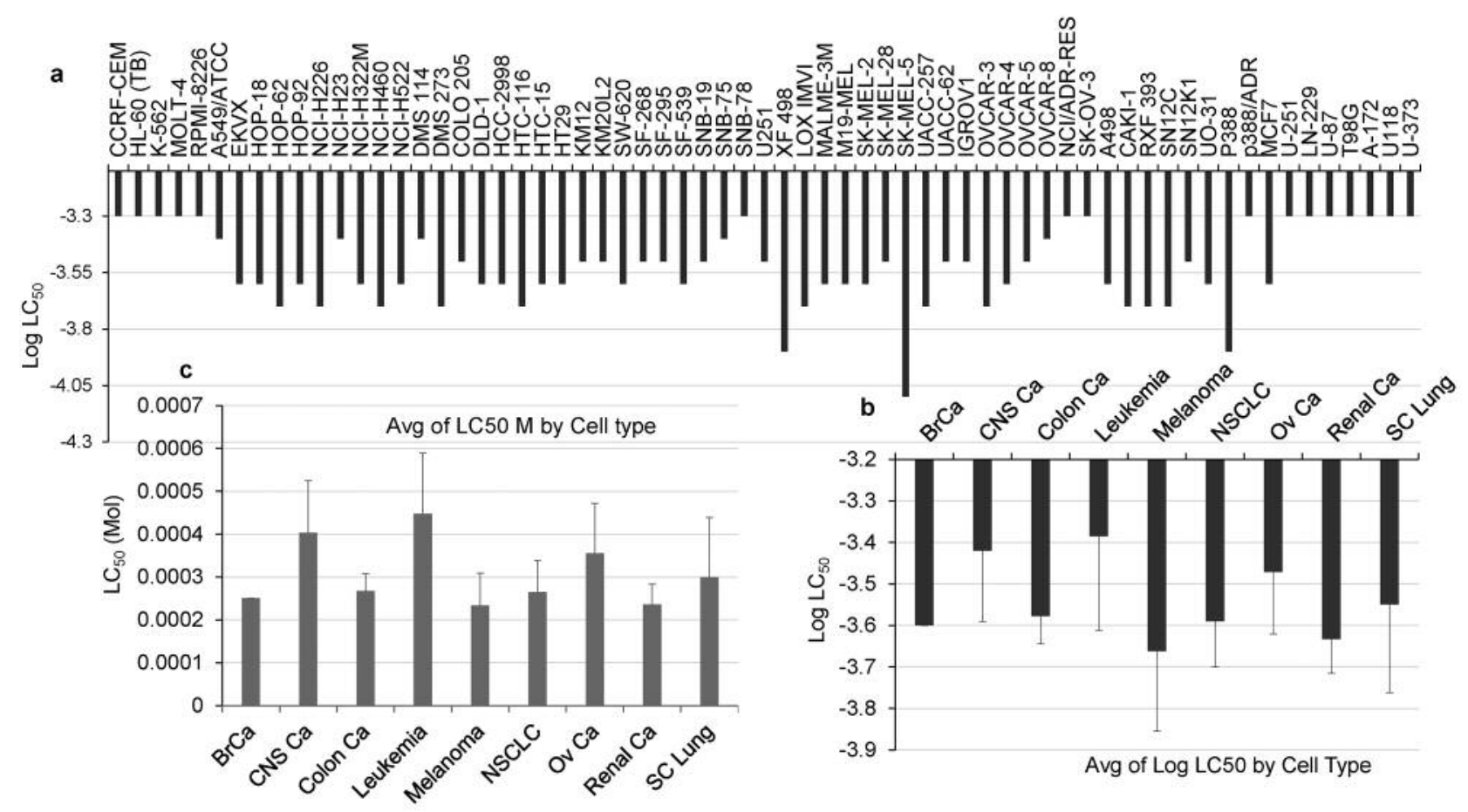

Figure 4. Mean graph of inhibitory effect of eucalyptol and the lethal concentration $50\left(L C_{50}\right)$ values (five dose response experiment). The data presented as $\log 10\left(L C_{50}\right)$ versus individual cell line. a. Data represented the $L C_{50}$ values for individual cell lines. $b$. Average log $L C_{50}$ values with the different cancer cell-type clusters. $c$. Different cancer cell type cluster versus $L C_{50}$ values.

Effect of eucalyptol and corresponding lethal concentration$50\left(L C_{50}\right)$ values for the different cell-line clusters. First, it is important to explain the different inhibitory parameters used in this study for anticancer drugs such as $\mathrm{GI}_{50}$, TGI and $\mathrm{LC}_{50}$. According to NCI nomenclature, the $\mathrm{IC}_{50}$ value is defined as the concentration that causes $50 \%$ growth inhibition and $\mathrm{GI}_{50}$ is the concentration of test drug where $100 \times\left(\mathrm{T}-\mathrm{T}_{0}\right) /(\mathrm{C}$ $\left.\mathrm{T}_{0}\right)=50$ (16). In this equation, $\mathrm{T}$ is the optical density of the test well after $48 \mathrm{~h}$ period of incubation with the test drug, $\mathrm{T}_{0}$ is the optical density at time zero, and $\mathrm{C}$ is the control optical density. The "50" is called the $\mathrm{GI}_{50} \operatorname{Prcnt}$, a T/C-like parameter that can have values from +100 to -100 . The $\mathrm{GI}_{50}$ measures the growth inhibitory power of the drug of interest. The TGI is the concentration of the test drug where $100 \times(\mathrm{T}-$ $\left.\mathrm{T}_{0}\right) /\left(\mathrm{C}-\mathrm{T}_{0}\right)=0$. Thus, the TGI signifies a cytostatic effect. The $\mathrm{LC}_{50}$, which signifies a cytotoxic effect, is the concentration of the drug where $100 \times\left(\mathrm{T}-\mathrm{T}_{0}\right) / \mathrm{T}_{0}=-50$. The control optical density is not used in the calculation of $\mathrm{LC}_{50}$. Next, the data for the inhibitory effect was extracted and the lethal concentration $-50\left(\mathrm{LC}_{50}\right)$ values of eucalyptol on various type of cancer cells were determined (Figures 3 and 4). The lethal concentration $\left(\mathrm{LC}_{50}\right)$ is the concentration of a chemical that kills 50 percent of the sample population under a certain study. The $\mathrm{LC}_{50}$ is usually applied to chemicals that are inhaled. Comparisons were made across the NCI- 60 cancer cell lines using the mean graph of five-dose eucalyptol (NSC6171) inhibitory activity in terms of $\mathrm{LC}_{50}$ values. The average $\mathrm{LC}_{50}$ over all cancer cell lines is $2.94 \times 10^{-4} \mathrm{M}$. The cell lines (CNS cancer panel, Figure 3B) showed a strong resistance towards eucalyptol. Leukemia cell lines (Figure $3 \mathrm{E})$ also showed a strong resistance to this drug. However, melanoma, renal and colon cancer cells showed sensitivity to this drug (Figures 3 and 4). Figure 4 represents individual cancer cells with corresponding $\log \mathrm{LC}_{50}$ values (eucalyptol concentrations, Figure 4A). The average of five-dose eucalyptol $\mathrm{LC}_{50}$ or $\log \mathrm{LC}_{50}$ values for different cancer types is also presented (averaging the values of individual cell lines of same type of cancer; Figure $4 \mathrm{~B}$ and C).

Compounds related with eucalyptol and ANXA7 found in NCI-60 ADS database by COMPARE analysis. Next, the other most relevant drug molecules that have the highest level Pearson correlation with the ANXA7 and eucalyptol were looked for. The COMPARE analysis was used to obtain the most significant anti-cancer drug molecules by seeding the ANXA7 and eucalyptol to set the algorithm. A complete list of drugs found based on $\mathrm{GI}_{50}$, TGI and $\mathrm{LC}_{50}$ are shown in Tables I-IV. The most significant and repeated drug 
<smiles>COc1cc(NS(C)(=O)=O)ccc1Nc1c2ccccc2nc2ccccc12</smiles>

Figure 5. The structure of the most common and significant compounds found by the COMPARE algorithm (a-f). The algorithm was set with different parameters such as inhibitory parameters $\left(G_{50}, T G I, L C_{50}\right)$ of eucalyptol along with ANXA7 expression in NCI-60 cancer cell lines panel. The different anticancer synthetic drugs found for this particular study are listed in the Tables I-IV.

structures are presented in the Figure 5. The highest-level correlation values were found with eucalyptol (NSC 6171), menogaril (NSC 269148), rapamycin (NSC 226080), Cyclopentenylcytosine (NSC 375575).

Validation of possible association of eucalyptol with ANXA7 induced metastatic castration-resistant prostate cancer cell death. The preliminary correlative associations were validated by carrying out a cell proliferation assay at different concentrations of ANXA7 and $\mathrm{LC}_{50}$ of $\mathrm{GI}_{50}(50 \%$ growth inhibition) concentration of eucalyptol and vice versa. It is important to mention that the prostate cancer cells are not sensitive to ucalyptol as ANXA7 expression is not increased by this drug. A time point was chosen where ANXA7 or eucalyptol by themselves did not have a significant effect. For in vitro experiments, DU145 cells $\left(1 \times 10^{6}\right)$ were plated and incubated with eucalyptol $(200 \mu \mathrm{M})$ for $3 \mathrm{~h}$ with gentle agitation. The cells were incubated for 6 $\mathrm{h}$ with adenovirus containing wild-type ANXA7 at various concentrations $(1,5$, or 10 multiplicity of infection (MOI)) after the addition of fresh medium containing $1 \%$ fetal bovine serum (Figure 6B). Cells incubated with the virus containing vector alone served as a control. In a second set of experiments, cells were first incubated with ANXA7 (adenovirus, MOI=1) for $3 \mathrm{~h}$ followed by a further $6 \mathrm{~h}$ incubation at various concentrations of eucalyptol (Figure $6 \mathrm{~A})$. Figure 6 shows a representative of two sets of experiments, since both yielded the same pattern. These results indicate that the synergistic action of ANXA7 and eucalyptol kills the metastatic prostatic cancer cells more efficiently than the single agent alone.

The gene expression profiling platform distinguishes the synergy between ANXA7 and response to eucalyptol. It was reasoned that significant changes in the ANXA7 signaling pathway with and without eucalyptol in prostate cancer may yield information on the biochemical mechanisms by which ANXA7 activates eucalyptol chemosensitivity, as well as hints to novel therapeutic approaches. The approach that was employed was to obtain gene expression profiles of eucalyptol or ANXA7 and compare it to that of their synergistic effect and to analyze the respective patterns on cDNA microarrays. 
Table I. The compounds are found by the COMPARE algorithm as correlating with eulacyptol function and GI50 end point. The settings were as follows: GI50 endpoint, Target Set: STANDARD_AGENTS_GI50. SEED: Eucalyptol (P-CINEOLE) NSC: 6171 Endpt:GI50 Exp Id: AVGDATA. SEED TYPE: NSC_FIVE_DOSE. The full chemical names of all these findings may be located at http://pubchem.ncbi.nlm.nih.gov. To find the all other corresponding information, DTP COMPARE AllCorr. Look up word: ANXA7_ter_0508.

\begin{tabular}{lccc}
\hline Rank & Compound & Correlation (r) & Cell lines \\
\hline 1 & Menogaril--NSC:S269148 hiConc:-4.0 & 0.568 & 55 \\
2 & Amonafide--NSC:S308847 hiConc:-3.6 & 0.564 & 55 \\
3 & Mitomycin C--NSC:S26980 hiConc:-4.6 & 0.559 & 55 \\
4 & MX2 HCl--NSC:S619003 hiConc:-4.6 & 0.545 & 55 \\
5 & VP-16 (etoposide)--NSC:S141540 hiConc:-3.0 & 0.54 & 55 \\
6 & m-AMSA (amsacrine)--NSC:S249992 hiConc:-3.6 & 0.529 & 55 \\
7 & Oxanthrazole--NSC:S349174 hiConc:-4.6 & 0.528 & 47 \\
8 & VM-26 (teniposide)--NSC:S122819 hiConc:-4.6 & 0.528 & 55 \\
9 & Oxanthrazole--NSC:S349174 hiConc:-3.6 & 0.527 & 55 \\
10 & m-AMSA (amsacrine)--NSC:S249992 hiConc:-4.6 & 0.526 & 45 \\
\hline
\end{tabular}

Table II. The compounds are found by the COMPARE algorithm as correlating with eucalyptol function and TGI end-point. Analysis parameters as follows: TGI endpoint TARGET SET: STANDARD_AGENTS_TGI SEED: P-CINEOLENSC:S6171 Endpt:TGI ExpId:AVGDATA hiConc:-3.3. SEED TYPE: NSC_FIVE_DOSE; ExpId: Avg Data.

\begin{tabular}{lccc}
\hline Rank & Compound & Correlation (r) & Cell lines \\
\hline 1 & Rapamycin--NSC:S226080 hiConc:-8.0 & 0.694 & 46 \\
2 & Amonafide--NSC:S308847 hiConc:-3.6 & 0.599 & 55 \\
3 & (Carboxyphthalato)platinum--NSC:S271674 hiConc:-4.0 & 0.589 & 55 \\
4 & ICRF-187--NSC:S169780 hiConc:-2.6 & 0.559 & 55 \\
5 & Hepsulfam--NSC:S329680 hiConc:-2.6 & 0.556 & 55 \\
6 & Oxanthrazole--NSC:S349174 hiConc:-3.6 & 0.553 & 54 \\
7 & Mitomycin C--NSC:S26980 hiConc:-4.6 & 0.552 & 55 \\
8 & Porfiromycin--NSC:S56410 hiConc:-3.0 & 0.55 & 55 \\
9 & Aclacinomycin A--NSC:S208734 hiConc:-3.6 & 0.531 & 55 \\
10 & Anguidine--NSC:S141537 hiConc:-7.0 & 0.518 & 47 \\
\hline
\end{tabular}

Table III. The compounds are found by the COMPARE algorithm as correlating with ANXA7 function (excluding eucalyptol) and GI50 end point. The settings were as follows: GI50 endpoint. TARGET SET: STANDARD_AGENTS_GI50. SEED: ANXA7 MICROARRAY_GENELOGIC_U133-MoltId: GC178578 Genecard: ANXA7.TargetSet: MOLTID_GC_SERIES_MICROARRAY_GENELOGIC_U133 SEED TYPE: MOL_TARG_GC_SERIES_NSC:S6171.

\begin{tabular}{lcll}
\hline Rank & Compounds & Correlation (r) & Cell lines \\
\hline 1 & Cyclopentenylcytosine--NSC:S375575 hiConc:-3.2 & 0.396 & 37 \\
2 & Maytansine--NSC:S153858 hiConc:-3.6 & 0.328 & 47 \\
3 & "o,p'-DDD"--NSC:S38721 hiConc:-3.9 & 0.318 & 60 \\
4 & Cyanomorpholino-ADR--NSC:S357704 hiConc:-8.0 & 0.3 & 59 \\
5 & Emofolin sodium--NSC:S139490 hiConc:-2.0 & 0.29 & 60 \\
6 & Triciribine phosphate--NSC:S280594 hiConc:-2.3 & 0.244 & 0.238 \\
7 & Rhizoxin--NSC:S332598 hiConc:-4.3 & 0.234 & 60 \\
8 & VP-16 (etoposide)--NSC:S141540 hiConc:-3.0 & 0.21 & 60 \\
9 & Triciribine phosphate--NSC:S280594 hiConc:-3.3 & \\
\hline
\end{tabular}

To assess the downstream targets of activated ANXA7 in prostate cancer cells, mRNAs were isolated from DU145 cells incubated with eucalyptol or ANXA7 alone or sensitized with eucalyptol for $3 \mathrm{~h}$ and incubated with ANXA7 for further $3 \mathrm{~h}$. The ATLAS ${ }^{\mathrm{TM}}$ cDNA expression cancer array was used to obtain the expression profiles. A comparison of the transcripts between different experimental paradigms was made using our GRASP algorithm (15). Table V shows the 
Table IV: The COMPARE algorithm to find the relevance of compounds correlated to ANXA7 function with TGI or LC50 endpoint. The parameters are as follows: TARGET SET: STANDARD_AGENTS TGISEED: ANXA7_MICROARRAY_GENELOGIC_U133 MoltId: GC178578 Genecard: ANXA7 TargetSet:MOLTID_GC_SERIES_MICROARRAY_GENELOGIC_U133 SEED TYPE:MOL_TARG_GC_SERIES

\begin{tabular}{lccc}
\hline Rank (for TGI) & Compounds & Correlation (r) & Cell lines \\
\hline 1 & VP-16 (etoposide)--NSC:S141540 hiConc:-3.0 & 0.275 & 60 \\
\hline Rank (for LC 50 ) & Compounds & Correlation (r) & Cell lines \\
\hline 1 & m-AMSA (amsacrine)--NSC:S249992 hiConc:-4.0 & 0.237 & 50 \\
2 & Caracemide--NSC:S253272 hiConc:-2.0 & 0.21 & 60 \\
\hline
\end{tabular}

Table V. Synergy between ANXA7 (A7) and response to eucalyptol (E) - showing only enhancement in the same direction as the A7 only effect. Combination is E3A3. Down-regulation (-Ve) or up-regulation (+Ve) indicated as the comparison to the ratio of A7 to E3A3.

\begin{tabular}{|c|c|c|c|c|c|}
\hline Name & GeneBank & E6 & A7 & E3A3 & E3A3 syn \\
\hline Ras homolog gene family, member A & L25080 & 0.962 & 0.899 & 0.478 & -1.88 \\
\hline Spectrin, beta, erythrocytic (inlcudes spherocytosis, clincialtype I) & J05500 & 1.095 & 0.938 & 2.056 & +1.88 \\
\hline Ras-related associated with diabetes & L24564 & 1.084 & 0.918 & 0.499 & -1.84 \\
\hline Mdm4, transformed $3 \mathrm{~T} 3$ cell double minute 4,53 binding protein (mouse) & AF007111 & 0.699 & 0.543 & 1.165 & +1.67 \\
\hline Cholecystokinin A receptor & L13605 & 0.67 & 0.762 & 1.235 & +1.62 \\
\hline Discs, large (Drosophila) homolog 3 (neuroendocrine-dlg) & U49089 & 0.754 & 0.785 & 0.47 & -1.6 \\
\hline CDK2-associated protein 1 & AF006484 & 0.814 & 0.75 & 1.288 & +1.58 \\
\hline Retinoblastoma-like 2(p130) & X74594 & 1.177 & 1.209 & 1.816 & +1.5 \\
\hline Matrix metalloproteinase 11(stromelysin 3) & X57766 & 0.482 & 0.495 & 0.322 & -1.5 \\
\hline Nuclear factor of kappa light polypeptide gene enhancer in B-cells $2(\mathrm{p} 49 / \mathrm{p} 100)$ & X61498 & 0.887 & 0.84 & 0.562 & -1.49 \\
\hline cyclin A2 & X51688 & 1.128 & 0.899 & 1.675 & +1.48 \\
\hline G protein-coupled receptor 4 & $\mathrm{U} 21051$ & 0.67 & 0.761 & 0.452 & -1.48 \\
\hline TRAF family member-associated NFKB activator & U59863 & 0.67 & 0.761 & 0.452 & +1.47 \\
\hline Vascular endothelial growth factor & M32977 & 0.986 & 0.939 & 1.446 & +1.47 \\
\hline Proline rich 2 & U03105 & 1.924 & 1.73 & 2.819 & +1.47 \\
\hline Fibroblast-growth factor receptor 3 (achondroplasia, thanatophoric dwarfism) & M58051 & 1.059 & 1.087 & 1.582 & +1.45 \\
\hline v-erb-b2 erythroblastic leukemia viral oncogene homolog 3 (avian) & M29366 & 0.892 & 0.918 & 0.613 & -1.45 \\
\hline Nuclear factor of kappa light polypeptide gene enhancer in B-cells inhibitor, alpha & M69043 & 0.602 & 0.714 & 1.039 & +1.45 \\
\hline Protein tyrosine phosphatase type IVA, member 1 & U48296 & 1.559 & 1.568 & 2.276 & +1.45 \\
\hline Baculoviral IAP repeat-containing 4 & U45880 & 0.74 & 0.694 & 1.06 & +1.43 \\
\hline CDC-like kinase 1 & L29222 & 1.075 & 0.98 & 1.537 & +1.43 \\
\hline Ras homolog gene family, member $\mathrm{E}$ & X95282 & 1.383 & 1.39 & 1.956 & +1.41 \\
\hline Monoglyceride lipase & U67963 & 1.323 & 1.422 & 0.941 & -1.41 \\
\hline Ribonucleotide reductase M2 polypeptide & X59618 & 1.319 & 1.352 & 1.899 & +1.4 \\
\hline
\end{tabular}

results of the microarray data analysis described in the Methods section and reveals the genes that are most affected by eucalyptol or ANXA7 alone or with ANXA7 plus eucalyptol. E6 is eucalyptol incubated for $6 \mathrm{~h}$, E3 is eucalyptol incubated for $3 \mathrm{~h}$, and A3 is Ad-wt-ANXA7 incubated for 3 h. 44 genes that could distinguish the effect of eucalyptol or ANXA7 alone and ANXA7 plus eucalyptol were found. The numbers mentioned in parenthesis below represent ratios reflecting up- or down-regulation in gene expression. According to the identified increase in cancer cell elimination, the synergistic effects of ANXA7 and eucalyptol resulted in concordant changes in gene expression profiles. Ras family members displayed the most frequent but not similar changes possibly reflecting their diverse roles in cell survival: Ras homolog gene family, member A (1.88; downregulation), Ras-related associated with diabetes (1.84; downregulation), and Ras homolog gene family, member E (1.41; up-regulation). Down-regulation (1.45) of v-erb-b2 erythroblastic leukemia viral oncogene homolog 3 (avian) but up-regulation (1.67) of the p53 binding protein Mdm4, transformed $3 \mathrm{~T} 3$ double cell minute 4 (mouse) were also concordant to the ANXA7 plus eucalyptol induced apoptosis 

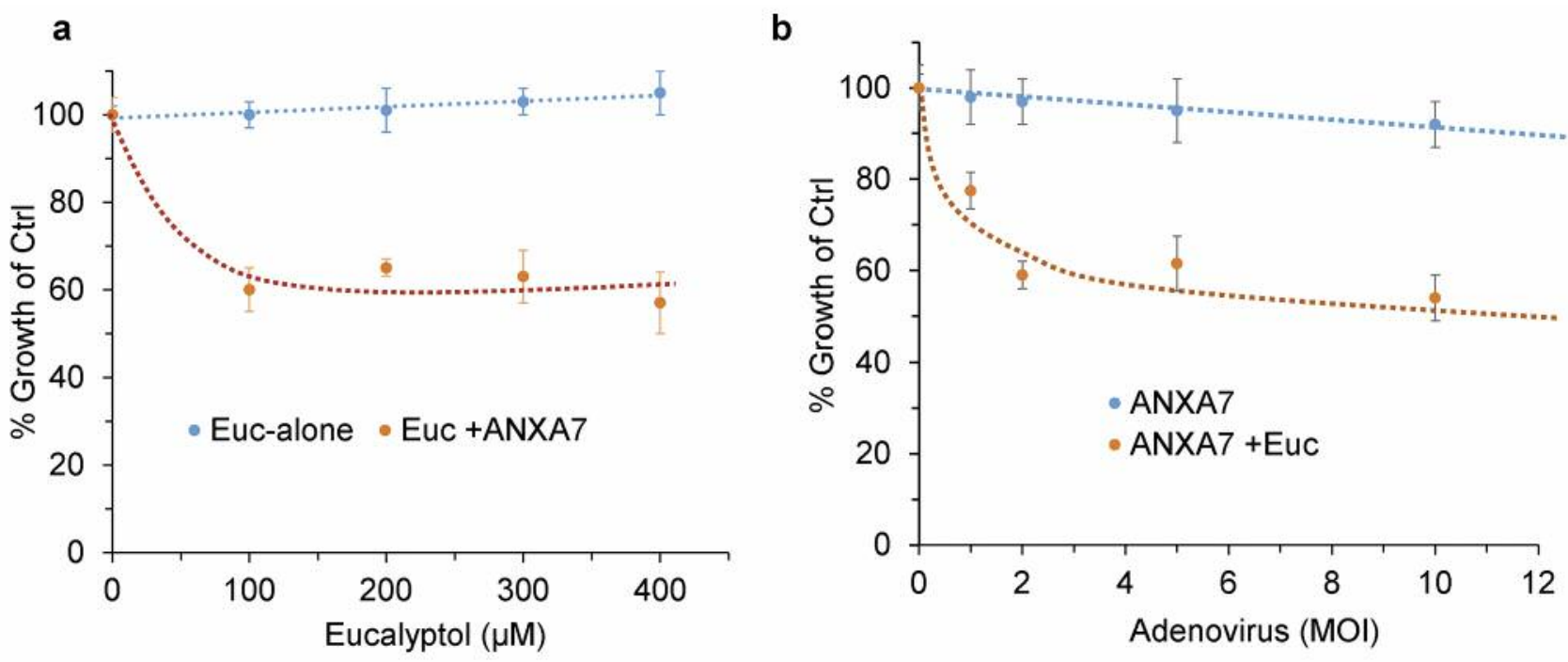

Figure 6. Effect of ANXA7 and eucalyptol on DU145 cell growth. a. The inhibitory effect of eucalyptol alone (blue line) and the synergetic effect of eucalyptol plus adenovirus expressed ANXA7 (red line) towards inducing the apoptotic pathway. In this experiment different concentrations of eucalyptol were incubated with the DU145 cells followed by the addition of ANXA7-expressing adenovirus (MOI 1). b. In this case the ANXA7expressing adenoviruses were incubated first and then a constant concentration of eucalyptol $(200 \mu M)$ was added. In both cases the results turned out the same. However, ANXA7 alone can slightly induce apoptosis, panel $b$, red line.

were observed. Some genes reflected predominant alterations in cell growth control (CDK2-associated protein 1, 1.58; CDC-like kinase 1, 1.43; cyclin A2, 1.48; up-regulation for all cases) and associated neoangiogenesis (vascular endothelial growth factor, 1.47; up-regulation) that was presumably reconstituted through the NF-kB pathway (nuclear factor of kappa light polypeptide gene enhancer 2 in B-cells (p49/p100), (1.49; down-regulation). Besides, the TRAF family member-associated NF-kB activator (1.47; upregulation) and nuclear factor of kappa light polypeptide gene enhancer in B-cells inhibitor, alpha, (1.45; up-regulation) are also induced in this pathway. Remarkably, monoglyceride lipase was among some of the genes responding to the combination ANXA7 with eucalyptol diversely (1.41 versus $\sim 1.4$ for ANXA7 and eucalyptol alone, for down-regulation and up-regulation respectively) that probably reflected associations of a monoterpene eucalyptol with lipid-relevant pathways (such as cholesterol derivatives isoprenoids) in cell survival. Thus, these results identify an ANXA7 dependent eucalyptol pathway that is mostly involved in cell survival and neoangiogenesis in prostate cancer cells.

The pathways associated with the expression of these genes were also analyzed (Figure 7). The gene expression data (Table V) was introduced into Ingenuity pathway analysis (IPA) software, and the different cancer signaling pathways associated with gene expression were obtained (panel-a); the associated biogenesis pathways (panel-b) and the induced metabolic pathways (panel-c).

\section{Discussion}

Although metastatic castration-resistant prostate cancer has long been associated with poor clinical outcome in cancer, only recently ANXA7 has been identified as the underlying mechanism (9). The castration-resistant prostate cancer cells are resistant to cytotoxic drugs due to their remarkably low rate of proliferation. Induction of apoptosis in sensitive tumor cells is the main mechanism of action of chemotherapy agents in human cancers. Our discovery, in this paper, that wtANXA7 sensitizes eucalyptol in metastatic castrationresistant prostate cancer cells may provide a new conceptual breakthrough. This is because activation of ANXA7 function can lead to increased apoptosis of tumor cells, since we have shown that ANXA7 is lost in castration-resistant prostate cancer and ANXA7 gene therapy suppresses the malignant properties of prostate cancer cells by a process involving $\mathrm{G}_{2}$ phase arrest followed by apoptosis (17). In this paper, ANXA7 was found to selectively sensitize eucalyptol to kill metastatic castration-resistant prostate cancer effectively and signaling pathways relevant for future drug design were identified. By providing a mechanism-based approach to complimentary cancer care, our studies could significantly alter the concepts that drive the fields of apoptotic signaling, ANXA7 signaling, chemosensitivity, cancer biology and complimentary therapy.

ANXA7 expression is completely lost in a high proportion of metastases (57\%) and local recurrences of castration- 
a
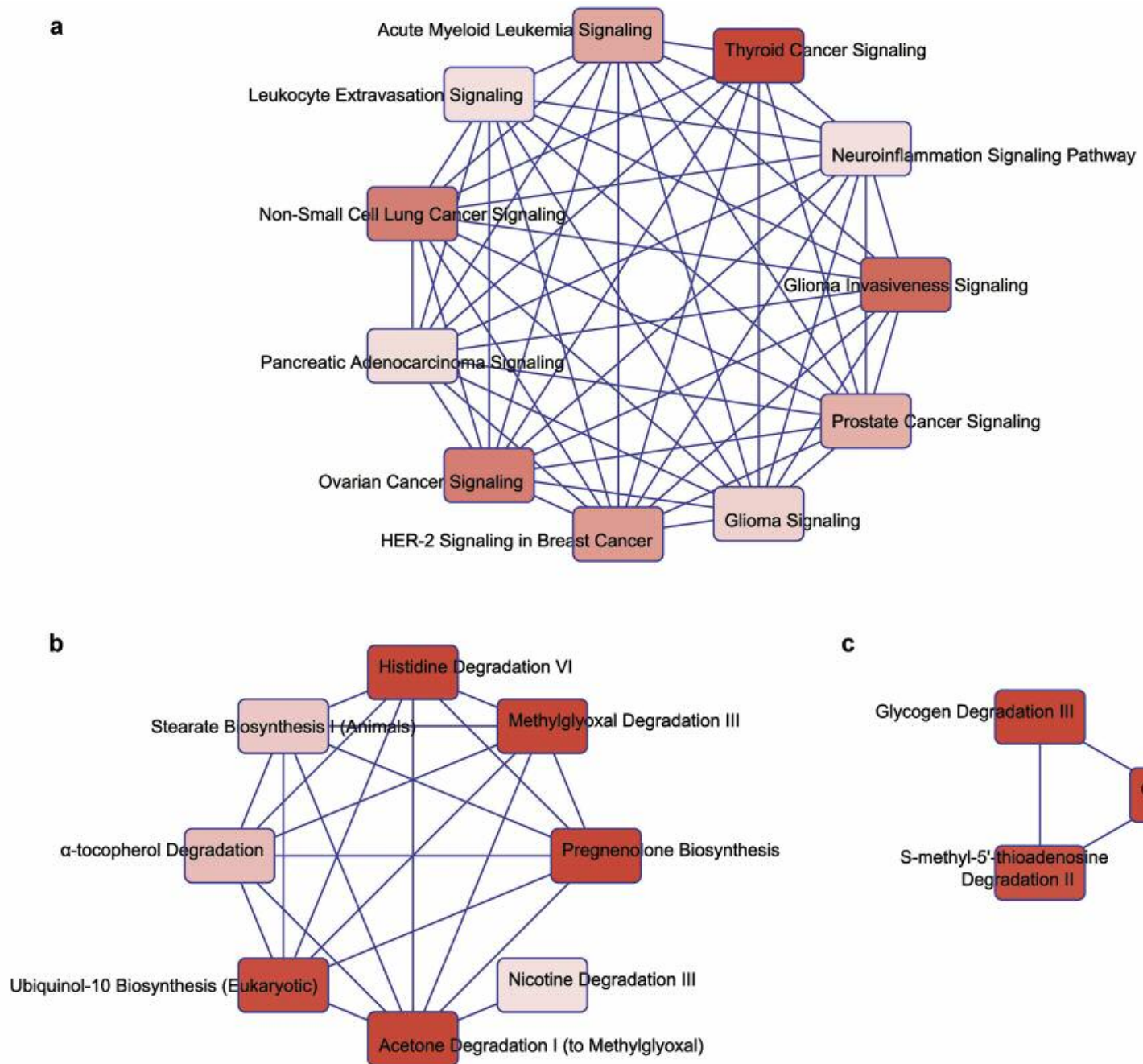

c

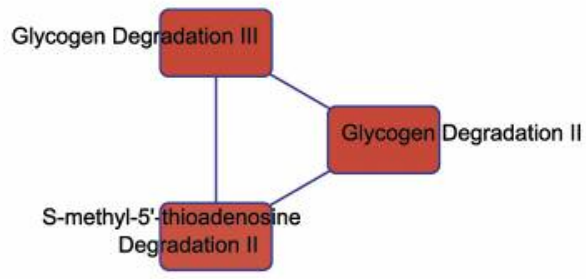

Figure 7. The signaling pathway analyses were performed based on the gene-expression data (associated with eucalyptol/ANXA7 axis) obtained through microarray experiment. The most significant microarray data were presented in the tabular form (Table-1). The gene-expression data were then analyzed through Ingenuity pathway analysis (IPA; https://www.qiagenbioinformatics.com/products/ingenuity-pathway-analysis/). a. This panel represented a series of different types of cancer associated with this gene-expression. $b$. This panel demonstrated the different biogenesis pathways regulating by the gene-expression associated with ANXA7/eucalyptol axis. c. Different metabolic pathways are induced by this gene expression.

resistant prostate cancer $(63 \%)$. Besides, our recent data indicated that in prostate cancer the ANXA7-mediated apoptotic pathway is cyclin E and FGF8 dependent (18). This data strongly suggest that the ANXA7 gene has clinical relevance for prostate cancer. By contrast, ANXA7 occurs at close to normal levels in benign prostate glands, high grade prostatic intraepithelial neoplasms (PIN), and stage T2 and T3/4 primary tumors (all in the range of $89-96 \%$ ) (5). The ANXA7-drug relationship for the naturally occurring eucalyptol exemplifies how variations in the protein levels relate to mechanisms of drug sensitivity and resistance. This is the first study to integrate ANXA7 expression and molecular pharmacology. Our results revealed that the overexpression of ANXA7 in the prostate cancer cell line correlated with enhanced sensitivity to eucalyptol.

Although monoterpenes were conventionally prescribed in chronic obstructive airway disorders mainly because of their secretolytic properties, eucalyptol also displayed antioxidant and cytokine inhibitory effects (19). Based on its effects on cytokine production, cytochrome $P-450$ system and lipid pathways (mostly isoprenoids and arachidonate cascade) that are relevant to innate immunity, cell growth, and survival as well as drug response, eucalyptol can be considered as a potential anticancer agent (20-22). To our knowledge, there are only a few reports referring to a therapeutic potential of eucalyptol in cancer treatment. Among various human cancer cells in vitro, 1,8- 
cineole induced apoptosis in the leukemia cell lines Molt 4B and HL60, but not in stomach cancer cells KATO III $(23,24)$. In a rat breast cancer model in vivo, dietary additions of monocyclic terpenes (d-limonene or (-)-menthol) resulted in significant inhibition of mammary carcinogenesis; however, bicyclic terpenes (including an oxygenated 1,8-cineole) had no significant chemopreventive activities at the tested dose levels (23). In a study on tamoxifen and gamma-linolenic acid (GLA) from borage oil, both of the other ingredients of the borage oil - ethanol and 1,8-cineole - enhanced permeation of tamoxifen and GLA across the pig skin supporting a plausibility of the simultaneous delivery of tamoxifen and GLA trans-cutaneously as an in vivo therapeutic approach in breast cancer (25). Eucalyptol also caused inhibition of testosterone- 6-betahydroxylation in human liver microsomes, presenting a therapeutic potential in the hormone modulation in cancer (26).

Also, some data on dietary agents in chemoprevention of carcinogenesis also suggested that certain monoterpenes (which are commonly used as flavoring agents and food additives) can be exploited for therapeutic purposes in cancer (27). Recognized as chemopreventive agents, monoterpenes (especially D-limonene and perillyl alcohol) have been demonstrated to induce phase II carcinogen-metabolizing enzymes, inhibit post-translational isoprenylation of small G-proteins including Ras oncogene as well as directly induce apoptosis in cancer cells (28-30). Also, limited efficacy of perillyl alcohol has been observed in clinical studies of patients with advanced prostate cancer (31). Some studies also suggested that monoterpenes can be more effective when used in combination with chemotherapeutic agents (32) or radiotherapy due to their capability of radiosensitizing prostate cancer cells through the augmentation of the Fas pathway (33).

The pathway analysis data demonstrated that the ANXA7mediated induction of eucalyptol's cytotoxicity is also similar with the gene-expression in thyroid cancer signaling pathway. Our unpublished data indicated that ANXA7 functions as tumor-suppressive gene and plays a critical role in the BRAF mediated chemoresistance in thyroid cancers.

Thus, additional studies are necessary to explore the therapeutic potential of eucalyptol and ANXA7, which are involved in the phospholipid aggregation and exocytosis, and in the management of cancer, especially in tumors from steroid hormone-regulated tissues such as breast and prostate that can be closely linked to the same lipid pathways as oxyand hydroxyl-substituted monoterpenes. In particular, preclinical studies on cytotoxic and other cancer-relevant effects of eucalyptol (alone or in combination with established anticancer drugs or tumor suppressors) in human cancer cells can reveal a promising therapeutic potential in carcinogenesis which so far has been a relatively underestimated field for cyclic monoterpenes.

Therefore, in the current study, a novel relationship between eucalyptol and ANXA7 expression was discovered in metastatic androgen-independent prostate cancer cells. Also, the combination of eucalyptol and ANX7 caused higher cell death than each one separately. Therefore, our data indicated that ANXA7 over-expression affects the cell survival signaling pathway as well as sensitizes cancer cells to eucalyptol by inducing apoptotic signaling to castration-resistant prostate cancer cells. Thus, determination of the effectiveness of eucalyptol in activating neoplastic ANXA7-mediated actions could rapidly lead to effective complimentary cancer therapy for castration-resistant prostate cancer.

\section{Conflicts of Interest}

The Authors declare no conflicts of interest regarding this study.

\section{Acknowledgements}

This work was supported by the grant to Dr. Meera Srivastava (DoD, DAMD17-03-1-0107).

\section{References}

1 Report: Cancer facts \& figures 2018. American Cancer Society, 2018.

2 Hill B and Kyprianou N: Sequencing hormonal ablation and radiotherapy in prostate cancer: A molecular and therapeutic prespective (review). Oncol Rep 9(6): 1151-1156, 2002.

3 Karantanos T, Corn PG and Thompson TC: Prostate cancer progression after androgen deprivation therapy: Mechanisms of castrate resistance and novel therapeutic approaches. Oncogene 32(49): 5501-5511, 2013.

4 Oh WK and Kantoff PW: Treatment of locally advanced prostate cancer: Is chemotherapy the next step? J Clin Oncol 17(11): 3664-3675, 1999.

5 Srivastava M, Bubendorf L, Srikantan V, Fossom L, Nolan L, Glasman M, Leighton X, Fehrle W, Pittaluga S, Raffeld M, Koivisto P, Willi N, Gasser TC, Kononen J, Sauter G, Kallioniemi OP, Srivastava S and Pollard HB: Anx7, a candidate tumor suppressor gene for prostate cancer. Proc Natl Acad Sci USA 98(8): 4575-4580, 2001.

6 Leighton X, Srikantan V, Pollard HB, Sukumar S and Srivastava M: Significant allelic loss of anx7region (10q21) in hormone receptor negative breast carcinomas. Cancer Lett 210(2): 239-244, 2004.

7 Paull KD, Shoemaker RH, Hodes L, Monks A, Scudiero DA, Rubinstein L, Plowman J and Boyd MR: Display and analysis of patterns of differential activity of drugs against human tumor cell lines: Development of mean graph and compare algorithm. J Natl Cancer Inst 81(14): 1088-1092, 1989.

8 Stinson SF, Alley MC, Kopp WC, Fiebig HH, Mullendore LA, Pittman AF, Kenney S, Keller J and Boyd MR: Morphological and immunocytochemical characteristics of human tumor cell lines for use in a disease-oriented anticancer drug screen. Anticancer Res 12(4): 1035-1053, 1992.

9 Weinstein JN, Kohn KW, Grever MR, Viswanadhan VN, Rubinstein LV, Monks AP, Scudiero DA, Welch L, Koutsoukos $\mathrm{AD}$, Chiausa AJ et al: Neural computing in cancer drug development: Predicting mechanism of action. Science 258(5081): 447-451, 1992. 
10 Weinstein JN, Myers TG, O'Connor PM, Friend SH, Fornace AJ, Jr., Kohn KW, Fojo T, Bates SE, Rubinstein LV, Anderson NL, Buolamwini JK, van Osdol WW, Monks AP, Scudiero DA, Sausville EA, Zaharevitz DW, Bunow B, Viswanadhan VN, Johnson GS, Wittes RE and Paull KD: An information-intensive approach to the molecular pharmacology of cancer. Science 275(5298): 343-349, 1997.

11 Scherf U, Ross DT, Waltham M, Smith LH, Lee JK, Tanabe L, Kohn KW, Reinhold WC, Myers TG, Andrews DT, Scudiero DA, Eisen MB, Sausville EA, Pommier Y, Botstein D, Brown $\mathrm{PO}$ and Weinstein $\mathrm{JN}$ : A gene expression database for the molecular pharmacology of cancer. Nat Genet 24(3): 236-244, 2000.

12 Ross DT, Scherf U, Eisen MB, Perou CM, Rees C, Spellman P, Iyer V, Jeffrey SS, Van de Rijn M, Waltham $M$, Pergamenschikov A, Lee JC, Lashkari D, Shalon D, Myers TG, Weinstein JN, Botstein D and Brown PO: Systematic variation in gene expression patterns in human cancer cell lines. Nat Genet 24(3): 227-235, 2000.

13 Deeken JF, Robey RW, Shukla S, Steadman K, Chakraborty AR, Poonkuzhali B, Schuetz EG, Holbeck S, Ambudkar SV and Bates SE: Identification of compounds that correlate with abcg2 transporter function in the national cancer institute anticancer drug screen. Mol Pharmacol 76(5): 946-956, 2009.

14 Creutz CE, Pazoles CJ and Pollard HB: Identification and purification of an adrenal medullary protein (synexin) that causes calcium-dependent aggregation of isolated chromaffin granules. J Biol Chem 253(8): 2858-2866, 1978.

15 Srivastava M, Eidelman O and Pollard HB: Pharmacogenomics of the cystic fibrosis transmembrane conductance regulator (cftr) and the cystic fibrosis drug cpx using genome microarray analysis. Mol Med 5(11): 753-767, 1999.

16 Monks A, Scudiero D, Skehan P, Shoemaker R, Paull K, Vistica D, Hose C, Langley J, Cronise P, Vaigro-Wolff A et al: Feasibility of a high-flux anticancer drug screen using a diverse panel of cultured human tumor cell lines. J Natl Cancer Inst 83(11): 757-766, 1991.

17 Torosyan Y, Dobi A, Naga S, Mezhevaya K, Glasman M, Norris C, Jiang G, Mueller G, Pollard H and Srivastava M: Distinct effects of annexin a7 and p53 on arachidonate lipoxygenation in prostate cancer cells involve 5-lipoxygenase transcription. Cancer Res 66(19): 9609-9616, 2006.

18 Bera A, Leighton X, Pollard H and Srivastava M: Cyclin e and fgf8 are downstream cell growth regulators in distinct tumor suppressor effects of anxa7 in hormone-resistant cancer cells of breast versus prostate origin. Trends Cancer Res, in press, 2018.

19 Worth H, Schacher C and Dethlefsen U: Concomitant therapy with cineole (eucalyptole) reduces exacerbations in copd: A placebo-controlled double-blind trial. Respir Res 10: 69, 2009.

20 Sampath S, Veeramani V, Krishnakumar GS, Sivalingam U, Madurai SL and Chellan R: Evaluation of in vitro anticancer activity of 1,8-cineole-containing n-hexane extract of callistemon citrinus (curtis) skeels plant and its apoptotic potential. Biomed Pharmacother 93: 296-307, 2017.
21 Duisken M, Benz D, Peiffer TH, Blomeke B and Hollender J: Metabolism of delta(3)-carene by human cytochrome p450 enzymes: Identification and characterization of two new metabolites. Curr Drug Metab 6(6): 593-601, 2005.

22 Duisken M, Sandner F, Blomeke B and Hollender J: Metabolism of 1,8-cineole by human cytochrome p450 enzymes: Identification of a new hydroxylated metabolite. Biochim Biophys Acta 1722(3): 304-311, 2005.

23 Russin WA, Hoesly JD, Elson CE, Tanner MA and Gould MN: Inhibition of rat mammary carcinogenesis by monoterpenoids. Carcinogenesis 10(11): 2161-2164, 1989.

24 Moteki H, Hibasami H, Yamada Y, Katsuzaki H, Imai K and Komiya T: Specific induction of apoptosis by 1,8 -cineole in two human leukemia cell lines, but not a in human stomach cancer cell line. Oncol Rep 9(4): 757-760, 2002.

25 Ho S, Calder RJ, Thomas $\mathrm{CP}$ and Heard CM: In vitro transcutaneous delivery of tamoxifen and gamma-linolenic acid from borage oil containing ethanol and 1,8-cineole. J Pharm Pharmacol 56(11): 1357-1364, 2004.

26 Miyazawa M, Shindo M and Shimada T: Roles of cytochrome p450 3a enzymes in the 2-hydroxylation of 1,4-cineole, a monoterpene cyclic ether, by rat and human liver microsomes. Xenobiotica 31(10): 713-723, 2001.

27 Shukla S and Gupta S: Dietary agents in the chemoprevention of prostate cancer. Nutr Cancer 53(1): 18-32, 2005.

28 Crowell PL: Prevention and therapy of cancer by dietary monoterpenes. J Nutr 129(3): 775S-778S, 1999.

29 Gould MN: Cancer chemoprevention and therapy by monoterpenes. Environ Health Perspect 105(Suppl 4): 977-979, 1997.

30 Karlson J, Borg-Karlson AK, Unelius R, Shoshan MC, Wilking $\mathrm{N}$, Ringborg $\mathrm{U}$ and Linder S: Inhibition of tumor cell growth by monoterpenes in vitro: Evidence of a ras-independent mechanism of action. Anticancer Drugs 7(4): 422-429, 1996.

31 Liu W and Rosazza JP: A soluble bacillus cereus cytochrome p450cin system catalyzes 1,4-cineole hydroxylations. Appl Environ Microbiol 59(11): 3889-3893, 1993.

32 Broitman SA, Wilkinson Jt, Cerda S and Branch SK: Effects of monoterpenes and mevinolin on murine colon tumor ct-26 in vitro and its hepatic "metastases" in vivo. Adv Exp Med Biol 401: 111-130, 1996.

33 Rajesh D, Stenzel RA and Howard SP: Perillyl alcohol as a radio-/chemosensitizer in malignant glioma. J Biol Chem 278(38): 35968-35978, 2003.
Received May 16, 2018

Revised June 7, 2018

Accepted June 15, 2018 